\title{
Platelet adhesiveness in diabetes mellitus
}

\author{
S. SHAW, G. D. PEGRUM, SYLVIA WOLFF, AND W. L. ASHTON \\ From the Departments of Haematology and Medicine, Charing Cross Hospital and Medical School, London
}

SYNOPSIS Platelet adhesiveness has been assessed on whole blood from a series of 34 diabetics and 50 control subjects using adenosine diphosphate (A.D.P.) and by adherence to glass microspherules (ballotini). Using both techniques it was possible to demonstrate a significant increase in platelet adhesiveness in the diabetic patients.

Increased platelet adhesiveness has been shown to occur in patients with ischaemic heart disease (MacDonald and Edgill, 1957). The close association of diabetes with an increased susceptibility to arterial disease suggested that changes in platelet stickiness might also occur in this disorder. Bridges, Dalby, Millar, and Weaver (1965) in a study of 22 diabetics found a significant increase in platelet adhesiveness to glass and also increased adhesiveness after administration of glucose in both healthy and diabetic persons. Ödegaard, Skålhegg, and Hellem (1964) were unable to show any increase in adhesiveness in 25 diabetic patients when passing whole blood through a column of glass beads, but found a marked increase in platelet adhesiveness in their diabetic subjects by adenosine diphosphate (A.D.P.)-induced adhesiveness in citrated platelet-rich plasma. We decided to study platelet adhesiveness in a series of diabetics using two whole-blood techniques, one dependnet on A.D.P., the other on adhesion to glass surfaces. The method of Eastham (1964), using A.D.P. was performed, together with a method using glass microspherules (Pegrum, Shaw, and Wolff, 1967).

The two procedures were carried out in parallel to give independent estimations of platelet adhesiveness. We regarded this as important in view of the finding of other workers and we were mindful that the various techniques used may measure different aspects of platelet stickiness (O'Brien, 1966).

\section{PATIENTS AND METHODS}

The 34 diabetic patients studied were being treated as out-patients by diet or diet and insulin. Their general health was reasonably good and none had clinical evidence of occlusive arterial disease. The group was not selected in any other way but by chance consisted of rather more males than females. The ages varied from 16 to 85 years. None of the patients was anaemic and Received for publication 27 February 1967. their platelet counts were within normal limits. The diabetic patients were matched with a series of controls who were selected from preoperative patients admitted to hospital with minor surgical conditions such as herniae and varicose veins. These patients were otherwise healthy and included only those with no family history of diabetes. Platelet adhesiveness was observed on 50 control patients with the same proportion of males to females as in the diabetic group and of the same age range.

Blood was taken with disposable syringes and no. 1 needles from a vein in the antecubital fossa, distended by gentle constriction immediately before puncture. The blood samples were collected in small batches from both patients and controls over a period of several weeks. Samples were collected between 2 and 4 p.m. on one or two afternoons a week and the adhesiveness was estimated within an hour. All experiments were carried out at room temperature. Polystyrene bottles $\left(2 \frac{1}{2}\right.$ in. $\times 1$ in.) were used in each test. Ethylenediamine tetra-acetic acid (E.D.T.A.) to give blood concentration of more than $4 \mathrm{mM}$ was used. The concentration of heparin was 100 units $/ \mathrm{ml}$. Platelet counts were carried out as described in the M.R.C. memorandum no. 32 (1955). Counts were performed in duplicate and the average taken and repeated if there were discrepancies greater than $15 \%$. In all the diabetics and matched controls the techniques were carried out by the observers without knowing from which group the samples were taken.

When using glass microspherules (ballotini) it was necessary to allow the spheres to settle before counting the platelets. This was achieved by allowing the container to stand for two minutes before counting from the particle-free upper layer. Control counts on blood allowed to stand in this way showed no significant difference from counts taken immediately after mixing.

Using Eastham's method $2 \mathrm{ml}$. of blood was added to $2.4 \mathrm{mg}$. E.D.T.A. and $2 \mathrm{ml}$. added to $2 \mathrm{mg}$. of heparin in separate polystrene bottles. Both samples were mixed on a Matburn wheel at 30 r.p.m. Adenosine diphosphate, $0.04 \mathrm{ml}$., was added to the heparinized sample. Adenosine diphosphate was prepared fresh from a stock solution of $25 \mathrm{mg}$. in $100 \mathrm{ml}$. of saline or stored in small aliquots at $-20^{\circ} \mathrm{C}$. for up to three months. The heparinized blood with added A.D.P. was returned to the mixer for 
exactly 30 minutes and then transferred to a second plastic bottle containing $2.4 \mathrm{mg}$. of E.D.T.A.; this was then mixed for a further 20 minutes and platelet counts were performed on both samples. At this stage samples can be left up to 24 hours at $4^{\circ} \mathrm{C}$. without significant change in platelet counts providing they are mixed for at least 20 minutes before counting. The adhesiveness was calculated as the percentage difference between the two counts.

Using glass microspherules (ballotini), $2 \mathrm{ml}$. of blood was added to $2.4 \mathrm{mg}$. of E.D.T.A. in a plastic bottle. This was mixed at 30 r.p.m. on a Matburn wheel and a sample removed for an initial platelet count. Then $3 \mathrm{~g}$. of ballotini (diameter between $0 \cdot 102$ and $0.124 \mathrm{~mm}$.) were added to the bottle which was returned to the wheel for exactly 35 minutes After standing for two minutes to allow the glass microspherules to settle and platelet count was performed. The adhesive platelet count was the difference in the initial number of platelets and the count following contact with the ballotini, expressed as a percentage.

\section{RESULTS}

USING ADENOSINE DIPHOSPHATE The results found in 34 diabetic patients were compared with the findings in 50 controls (Fig. 1). The mean adherence in the control group was $60.5 \%$ with a standard deviation of $7.2 \%$. We took as a normal range of platelet adhesiveness twice the standard deviation from the average $(46-75 \%)$. Two control subjects had adhesiveness outside this range; one male aged over 70 years was below and one male aged 23 years was above. Twenty of the diabetic patients out of the total of 34 had an adhesiveness above the upper limit of our normal range; none of the diabetics had levels below the normal range. The average mean adhesiveness for all the diabetic patients was $75.7 \%$ with a standard deviation of $10.5 \%$. Comparing the two groups using Gossets t test, there is a highly significant increase in platelet adherence $(P<0.001)$ in the diabetic patients.

USING ADHESION TO GLASS Twenty-five diabetic patients were studied and the results compared with the findings in 50 controls (Fig. 2). The mean adhesiveness using this technique was $53.4 \%$ with a standard deviation of $6.9 \%$. We took as our normal range of platelet adhesiveness twice the standard deviation from the average mean 39.5-67\%. Two observations in our control group were outside this range, one in a 57-year-old woman was above and one in a 75-year-old man was below. Twelve of the 25 diabetic patients had an adhesiveness above this range and one fell below the normal range. The mean adhesiveness in diabetics was $65.5 \%$ with a standard deviation of $11.5 \%$. Comparing the diabetic group

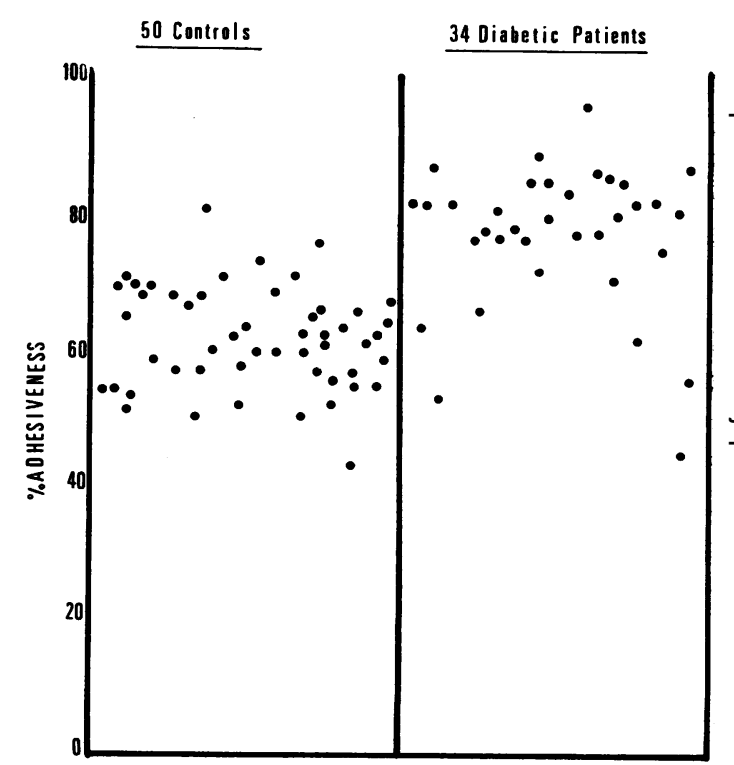

FIG. 1. Results with A.D.P. in 50 controls and 34 diabetics. Average value in controls was $60.5 \%$ and in diabetics $75.7 \%(P=<0.001$, highly significant $)$.

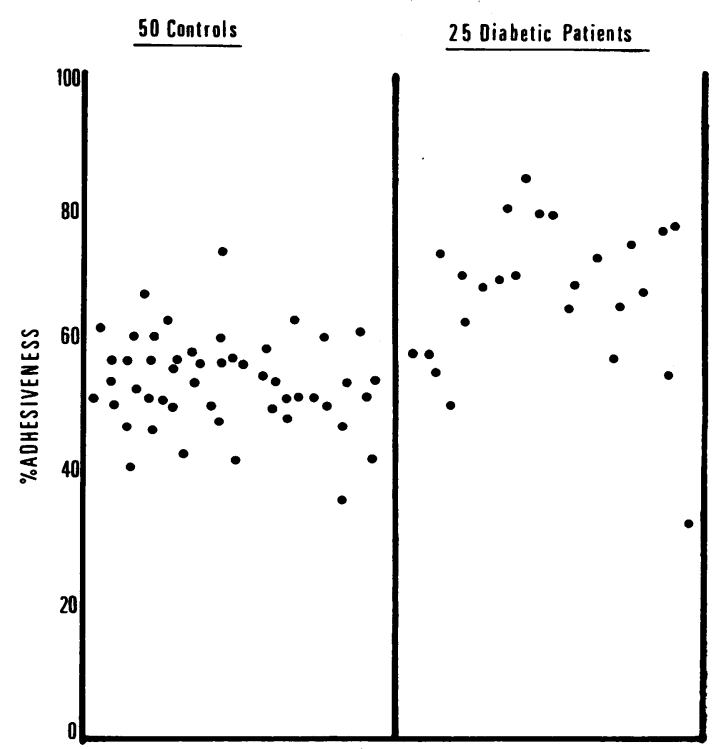

FIG. 2. Results with ballotini in 50 controls and 25 Фृ diabetics. Average value in controls was $52.7 \%$ and in diabetics $65.6 \%(P=<0.001$, highly significant $)$. 
with the controls there is a significantly increased platelet adhesiveness $(P<0.001)$ in diabetics.

\section{DISCUSSION}

The results presented here using whole blood demonstrate a marked increase in platelet adhesiveness in diabetic patients using both A.D.P. and adhesion to glass. This finding is in agreement with the work of Bridges et al. (1965) but differs from the observations of Ödegaard et al. (1964) who found no increased adhesion of platelets using whole blood from diabetics. In our experiments the contact with glass surfaces was considerably longer than in the experiments by Ödegaard and his colleagues, and this may account for the different findings. They considered the lack of change in adhesiveness to be due to a decreased release of A.D.P. from the red cells. It is likely that other factors as well as A.D.P. are concerned in adhesion, including collision factors between particles. We may therefore expect differences to be evident between the various experimental methods used to study platelet adhesiveness. Comparisons should probably only be made using exactly similar techniques.

In previous studies (Pegrum et al., 1967) we found that platelet adhesiveness was independent of age but there was a possible association with the sex of the individual. We therefore included in our control group the same proportion of males to females as in the diabetics; we also made observations using the same age range in both groups. The experimental errors inherent in the differential platelet counts are common to both groups and should not influence the results. We were unable to obtain standard conditions with regard to meals, smoking, exercise, or the exact time of venesection. However, batches of controls and diabetics were taken together and nearly all the samples were collected between 2 and 4 p.m. on any given day. Platelet adhesiveness has been shown to be influenced by the level of blood glucose. Increased adhesiveness has been observed after a glucose load (Bridges et al., 1965) and decreased adhesiveness following the rise associated with smoking (Murchison and Fyfe, 1966). Hellem (1960) was unable to show any significant change in adhesion to glass with physiological variations in blood glucose. No direct relationship between blood glucose levels and adhesiveness has been demonstrated and we thought it unlikely that the blood glucose level would have influenced our results. It seems probable that changes in the composition of plasma fatty acids is the most likely link between increased levels of platelet adhesiveness found in the diabetics and the associated susceptibility to arterial disease with increased risk of myocardial infarction. Increased adhesiveness has been shown to occur in myocardial infarction (MacDonald and Edgill, 1957) in which change in fatty acids occurs (Kerr, Pirrie, MacAuley, and Bronte-Stewart, 1965) but Ödegaard et al. (1964) were unable to find a correlation between adhesiveness and plasma cholesterol or total lipid values.

This aspect would seem worthy of further study to see if a direct association exists between diabetes with increased platelet adhesiveness and alterations of fatty acids with increased risk of occlusive arterial disease.

We are grateful to the Clinical Research Sub-Committee, Charing Cross Hospital, for financial support. Professor H. E. de Wardener kindly allowed us access to his patients. We also thank Miss P. Turnbull of the Photographic Department of Charing Cross Hospital for the photographs.

\section{REFERENCES}

Bridges, J. M., Dalby, M., Millar, J. H. D., and Weaver, J. A. (1965). Lancet, 1, 75.

Eastham, R. D. (1964). J. clin. Path., 17, 45.

Hellem, A. J. (1960). Scand. J. clin. Lab. Invest., 12, suppl. 51.

Kerr, J. W., Pirrie, R., MacAulay, I., and Bronte-Stewart, B., (1965). Lancet, 1, 1296.

McDonald, L., and Edgill, M. (1957). Ibid., 2, 457.

Murchison, L., and Fyfe, T. (1966). Lancet, 2, 182.

O'Brien, J. R., (1966). Ann. Rev. Med., 17, 275.

Odegaard, A. E., Skålhegg, B. A., and Hellem, A. J. (1964). Thrombos. Diathes. haemorrh. (Stuttg.), 11, 27.

Pegrum, G. D., Shaw, S., and Wolf, S. E. (1967). J. clin. Path., 20, 65 . 